\title{
ON ISOMETRIC EQUIVALENCE OF CERTAIN VOLTERRA OPERATORS
}

\author{
G. K. KALISCH ${ }^{1}$
}

The purpose of this paper is to extend the results of $\S 4$ of the author's paper [3, referred to as V] to $L_{p}[0,1]$ for all $p$ such that $1<p<\infty$. In general we shall use the notation and definitions of $\mathrm{V}$, except that the functions considered here are of the form

(1) $F(x, y)=(y-x)^{m-1} a G(x, y)$ where $\left\{\begin{array}{l}m \text { is a positive integer, } \\ |a|=1, \\ G(x, x)>0\end{array}\right.$

otherwise, as in $\mathrm{V}$, the complex valued function $G(x, y)$ is continuously differentiable. The only difference from $\mathrm{V}$ is the presence of the constant $a$ which affects the proof of Theorem 2 of V. A version of that theorem in the more general case where $a$ is an arbitrary constant of absolute value 1 will be published elsewhere [4]. All other theorems and proofs of $\mathrm{V}$ remain valid. The class $D$ of functions with which we are principally concerned may be described as follows: the functions $F$ are of the general form (1) where, in addition, $G$ and $m$ satisfy any one of the following: (A) $G$ is analytic in a suitable region and $m$ is an arbitrary positive integer (see Lemma 4 of $\mathrm{V}$ ); (B) $G(x, y)=G(y-x)$ where $G(0) \neq 0$ and $G \in C^{2}$ in a neighborhood of $y=x$ and otherwise $G(t) \in L_{1}[0,1]$ and $m$ is an arbitrary positive integer; (C) $G \in C^{2}$ and $m=1$. One very important property of the operators $T_{F}$ for $F \in D$ is the fact (see Theorem 3 of $\mathrm{V}$ ) that their only reducing manifolds are the subspaces $L_{p}[0, c]$ of $L_{p}[0,1]$ for all $c \in[0,1]$ (see also $[2 ; 5$ and 6$]$ ). This property is crucial for the establishment of unitary invariants (in the case $p=2$ ) of the operators $T_{F}$ in $\S 4$ of V. As is usual, we define $q$ by $1 / p+1 / q=1$.

Two continuous linear transformations $T_{1}$ and $T_{2}$ mapping $L_{p}[0,1]$ into itself are called isometrically equivalent if there exists an isometry $U$ of $L_{p}[0,1]$ onto itself such that $T_{1}=U T_{2} U^{-1}$ (regarding isometries for $p \neq 2$, see, e.g., $[1$, p. 178]; the considerations of the present paper are valid without this restriction). Two preliminary lemmas are needed in order to extend some results on Hilbert spaces and spectral theory to general $p$. We shall use the following notation: $M_{a}$ is the operator "multiplication by the characteristic function $c_{a}(x)$ of the

Received by the editors February 26, 1960.

1 The author was supported in part by the United States Air Force under Contract No. AF 49(638)-64 and by a grant from the National Science Foundation. 
interval $[0, a]$ "; similarly $M_{S}$ is the operator "multiplication by the characteristic function $c_{S}(x)$ of the subset $S$ of $[0,1]$." We shall occasionally write $E_{a}$ instead of $M_{a}$.

Lemma 1. Let $T_{0}$ be an idempotent bounded linear transformation of $L_{p}[0,1]$ into itself whose range is $L_{p}[0, a]$ for some $a \in(0,1]$. Then $\left\|T_{0}\right\|=1$ if and only if $T_{0}=M_{a}$.

Proof. Since $\left\|M_{a}\right\|=1$ is obvious, we turn to the converse. Let $N_{1}=L_{p}[0, a]$ and $N_{2}=L_{p}[a, 1]$. Then $T_{0}-M_{a}=T$ maps $N_{1}$ into 0 and $N_{2}$ into $N_{1}$; we have $T_{0}=M_{a}+T$. We wish to show that $T=0$. Suppose that $T \neq 0$; let $b=\|T\| \neq 0$. Find a positive real number $e$ such that

$$
\left(1+b^{q}\right)^{1 / q}-e \frac{b^{-1}+b^{q / p}}{\left(1+b^{q}\right)^{1 / p}}>1 ;
$$

now determine $f_{2} \in N_{2}$ such that $\left\|T f_{2}\right\|_{p} \geqq(b-e)\left\|f_{2}\right\|_{p}$ where

$$
\left\|f_{2}\right\|_{p}^{p}=\frac{b^{q}}{1+b^{q}} .
$$

Let $f_{1}=b^{-a} T f_{2} \in N_{1}$ and let $f=f_{1}+f_{2}$. Then $T_{0} f=f_{1}+T f_{2}=\left(b^{-q}+1\right) T f_{2}$. Since $f_{i} \in N_{i}(i=1,2)$ we have $\|f\|_{p}^{p}=\left\|f_{1}\right\|_{p}^{p}+\left\|f_{2}\right\|_{p}^{p}$ and

$$
\left\|f_{1}\right\|_{p}^{p}=b^{-p q} b^{p}\left\|f_{2}\right\|_{p}^{p}=\frac{b^{-p q} b^{p} b^{q}}{1+b^{q}}=\frac{1}{1+b^{q}} .
$$

Therefore

$$
\|f\|_{p}^{p}=\left\|f_{1}\right\|_{p}^{p}+\left\|f_{2}\right\|_{p}^{p} \leqq \frac{1}{1+b^{q}}+\frac{b^{q}}{1+b^{q}}=1 .
$$

On the other hand,

$$
\begin{aligned}
\left\|T_{0}\right\|_{p} & =\left\|f_{1}+T f_{2}\right\|_{p}=\left(b^{-q}+1\right)\left\|T f_{2}\right\|_{p} \\
& \geqq\left(b^{-q}+1\right)(b-e)\left\|f_{2}\right\|_{p} \\
& =\frac{\left(b^{-q}+1\right)(b-e) b^{q / p}}{\left(1+b^{q}\right)^{1 / p}} \\
& =\frac{\left(b^{-q}+1\right) b^{q}}{\left(1+b^{q}\right)^{1 / p}}-e \frac{b^{-1}+b^{q / p}}{\left(1+b^{q}\right)^{1 / p}} \\
& =\left(1+b^{q}\right)^{1 / q}-e \frac{b^{-1}+b^{q / p}}{\left(1+b^{q}\right)^{1 / p}} \\
& >1
\end{aligned}
$$


by the choice of $e$, see (2). But this contradicts $\left\|T_{0}\right\|=1$ so that $b$ and hence $T$ must be zero, i.e., $T_{0}=M_{a}$, and the proof of the lemma is complete.

The following lemma shows that the projections $E_{a}$ for all $a \in[0,1]$ generate a maximal abelian algebra in the algebra of all bounded linear transformations of $L_{p}[0,1]$ into itself not only for $p=2$ but indeed for all $p$ considered in this paper.

LEMMA 2. Let $T$ be a bounded linear transformation mapping $L_{p}[0,1]$ into itself and suppose that $T E_{a}=E_{a} T$ for all $a \in[0,1]$. Then there exists a bounded measurable function $f$ such that $T=M_{f}$ (= "multiplication by f").

Proof. Let $e=e(x)$ be the function identically equal to 1 and let $f(x)=(T e)(x)$. We shall show that $f$ is essentially bounded and that $T=M_{f}$. If $g$ is a simple function: $g(x)=\sum_{j} a_{j} c_{S(j)}(x)$, then $T g$ $=\sum_{j} a_{j} T c_{S(j)}$; but $c_{S}(x)=\left(M_{S} e\right)(x)$ so that $\left(T c_{S}\right)(x)=\left(T M_{S} e\right)(x)$ $=\left(M_{S} T e\right)(x)=\left(M_{S} f\right)(x)=f(x) c_{S}(x)$ since our hypothesis implies that $T$ commutes not only with all $E_{a}=M_{a}$ but also with all relevant $M_{S}$. Therefore $(T g)(x)=f(x) g(x)$ for all simple $g$. The boundedness of $T$ implies that $\|T g\|_{p}=\|f g\|_{p} \leqq\|T\|_{p}\|g\|_{p}, \int_{0}^{1}|f(x) g(x)|{ }^{p} d x \leqq\|T\|_{p}^{p}\|g\|_{p}^{p}$ for all simple $g$. Hence $|f|^{p}$ and $|f|$ are essentially bounded and $(T g)(x)$ $=f(x) g(x)$ for all $g \in L_{p}[0,1]$.

If $s=s(t)$ is a monotone increasing function defined on $[0,1]$ such that $s(0)=0$ and $s(1)=1$, we write $U_{s}=M_{\left(s^{\prime}\right)^{1 / p} S_{s}}$ where we use the notation of $\mathrm{V}$. If $s(t)$ is absolutely continuous with an inverse function of the same kind, then $U_{s}$ as a linear transformation of $L_{p}[0,1]$ into itself is an isometry onto.

THEOREM 1. Let $T_{F_{1}}$ and $T_{F_{2}}$ be two continuous linear transformations of $L_{p}[0,1]$ into itself whose only reducing manifolds are the subspaces $L_{p}[0, c]$ of $L_{p}[0,1]$ for all $c \in[0,1]$, such as, for example, the transformations $T_{F}$ for $F \in D$. Then if $T_{F_{1}}$ is isometrically equivalent to $T_{F_{2}}=U T_{F_{1}} U^{-1}$, there exist: (a) a measurable function $h(x)$ defined on $[0,1]$ such that $|h(x)| \equiv 1$; (b) a strictly monotone absolutely continuous function $s(x)$ defined on $[0,1]$ such that $s(0)=0$ and $s(1)=1$ with an inverse function of the same kind. We have $U=M_{h} U_{s}$. The functions $F_{1}$ and $F_{2}$ are then related by the equation

$$
F_{2}(x, y)=\frac{h(x)}{h(y)}\left(s^{\prime}(x)\right)^{1 / p}\left(s^{\prime}(y)\right)^{1 / q} F_{1}(s(x), s(y)) .
$$

If conversely two functions $F_{1}$ and $F_{2}$ are related by (3) where the functions $h(x)$ and $s(x)$ are defined as in (a) and (b) above, then $T_{F_{1}}$ is isometrically equivalent to $T_{F_{2}}=U T_{F_{1}} U^{-1}$ where $U=M_{h} U_{\text {. }}$. 
Proof. Suppose first that $T_{F_{2}}=U T_{F_{1}} U^{-1}$. Since both linear transformations have as their only reducing manifolds the spaces $L_{p}[0, c]$ for all $c \in[0,1]$, we can conclude that $U E_{t} U^{-1}=F_{r(t)}$ where $F_{r(t)}$ is idempotent with range $L_{p}[0, r(t)]$; therefore $r(t)$ is increasing and satisfies the equations $r(0)=0$ and $r(1)=1$. Since $\left\|E_{t}\right\|=1$ and $U$ is an isometry, we have $\left\|F_{r(t)}\right\|=1$ for all positive $r(t)$. Lemma 1 now implies that $F_{r(t)}=E_{r(t)}=U E_{t} U^{-1}$. To show that $r$ is absolutely continuous and strictly increasing, we consider for $g \in L_{q}[0,1]$ the expression $\left(E_{r(t)} f, g\right)=\left(U E_{t} U^{-1} f, g\right)=\left(E_{t} f_{1}, g_{1}\right)$ where $f_{1}=U^{-1} f$ and $g_{1}=U^{*} g$; the linear transformation $U^{*}$ is the adjoint of $U$ (acting in $\left.L_{q}[0,1]\right)$. If $f=g=1$ then $r(t)=\int_{0}^{t} f_{1}(x) g_{1}(x) d x$. This shows that $r(t)$ is absolutely continuous. If $f_{1}=g_{1}=1$ then $t=\int_{0}^{(r) t} f(s) g(x) d x$. This shows that $r(t)$ is strictly increasing; the inverse function $s(t)$ of $r(t)$ has the same properties. It is easy to verify that $U_{r}^{-1} E_{t} U_{r}=E_{r(t)}$; this equation together with the equation $U E_{t} U^{-1}=E_{r(t)}$ implies that $U_{r} U$ commutes with all $E_{t}$. Lemma 2 then implies that $U_{r} U=M_{k}$ where $k=k(x)$ is a bounded measurable function. Since $U_{r} U$ is an isometry, the function $k(x)$ must satisfy $|k(x)| \equiv 1$; we have $U=U_{r}^{-1} M_{k}$. The functions $s(x)$ and $h(x)=k(s(x))$ are the functions whose existence was asserted by the theorem; a simple calculation shows that $U=U_{r}^{-1} M_{k}=M_{h} U_{s}$ as promised. It is now an easy matter to verify (3); the computation needed for this purpose is similar to that needed to establish the converse of the theorem.

We state next the analog of Theorem 5 of $\mathrm{V}$; the formulas and proof are changed due to the presence of the constant $a$ in our present context, and to the arbitrariness of $p$.

Theorem 2. Let $F(x, y)=(y-x)^{m-1} a G(x, y)$ be of form (1) where $G \in C^{1}$ in a neighborhood of $y=x$ and let $T_{F}$, considered as a linear transformation of $L_{p}[0,1]$ into itself, have as its only reducing manifolds the spaces $L_{p}[0, c]$ for all $c \in[0,1]$, as is the case if $F \in D$. Then $T_{F}$ is isometrically equivalent to a unique $T_{F_{1}}=U T_{F} U^{-1}$ where $F_{1}$ and $G_{1}$ satisfy the following:

$$
\begin{aligned}
F_{1}(x, y) & =(y-x)^{m-1} a G_{1}(x, y), \\
G_{1}(x, x) & =c=\left(\int_{0}^{1}(G(u, u))^{1 / m} d u\right)^{m}>0, \\
\operatorname{Im}\left(G_{1 x}(x, x)\right) & =\operatorname{Im}\left(G_{1 y}(x, x)\right)=0 .
\end{aligned}
$$

This is achieved by setting $U=M_{h} U_{r}^{-1}$ where

$$
r(t)=(1 / c)^{1 / m} \int_{0}^{t}(G(u, u))^{1 / m} d u
$$


The function $h(x)$ is determined by defining

$$
F_{0}(x, y)=(y-x)^{m-1} a G_{0}(x, y)
$$

by $T_{F_{0}}=U_{r}^{-1} T_{F} U_{r}$ where $G_{0}=H_{0}+i K_{0}$ for real $H_{0}$ and $K_{0}$ and setting $h(x)=\exp \left((-i / c) \int_{0}^{x} K_{0 x}(u, u) d u\right)$.

Proof. If $r$ and $h$ are defined as described and if we set $T_{F_{1}}$ $=\left(M_{h} U_{r}^{-1}\right) T_{F}\left(M_{h} U_{r}^{-1}\right)^{-1}$ then $F_{1}$ does indeed satisfy (4). Thus every $T_{F}$ is isometric with $T_{F_{1}}$, where $F_{1}$ has form (4). To show uniqueness, suppose that $F_{i}=(y-x)^{m_{i}-1} a_{i} G_{i}(i=1,2)$ are both of form (4) and that $T_{F_{1}}$ is isometrically equivalent to $T_{F_{2}}=U T_{F_{1}} U^{-1}$. Then (3) implies that $F_{1}$ and $F_{2}$ are related by the following equation:

$$
\begin{aligned}
& (y-x)^{m_{2}-1} a_{2} G_{2}(x, y) \\
& =\frac{h(x)}{h(y)}\left(s^{\prime}(x)\right)^{1 / p}\left(s^{\prime}(y)\right)^{1 / q}\left(\frac{s(y)-s(x)}{y-x}\right)^{m_{1}-1}(y-x)^{m_{1}-1} a_{1} G_{1}(s(x), s(y)),
\end{aligned}
$$

where the functions $h$ and $s$ are as in (a) and (b) of Theorem 2. On letting $y-x$ approach zero we see that $m_{1}=m_{2}=m$ and that

$$
a_{2} G_{2}(x, x)=\left(s^{\prime}(x)\right)^{m} a_{1} G_{1}(s(x), s(x))
$$

since $1 / p+1 / q+m-1=m$. Equation (5) implies that $a_{1}=a_{2}=a$ since $G_{i}(x, x)=c_{i}>0$; we next observe that (5) also implies that $s(x) \equiv x$ and that $c_{1}=c_{2}=c$. Equation (3) now reduces to $G_{2}(x, y)$ $=(h(x) / h(y)) G_{1}(x, y)$ or

$$
h(y)\left(H_{2}(x, y)+i K_{2}(x, y)\right)=h(x)\left(H_{1}(x, y)+i K_{1}(x, y)\right),
$$

if we write $G_{j}=H_{j}+i K_{j}$ for real $H_{j}$ and $K_{j}(j=1,2)$. Our hypotheses imply that $H_{j}(x, x)=c$ and that $K_{j}(x, x)=K_{j x}(x, x)=K_{j y}(x, x)$ $=0(j=1,2)$ and also that $h(x)=\exp (i k(x))$ for real $k(x)$ is differentiable. Differentiation of (6) and setting $x=y$ yields $h(x) H_{2 x}(x, x)$ $=c h^{\prime}(x)+h(x) H_{1 x}(x, x)$ so that $h^{\prime} / h=i k^{\prime}=1 / c\left(H_{2 x}(x, x)-H_{1 x}(x, x)\right)$. But the last expression is real so that $k^{\prime}=0$, and $h$ is constant. We finally arrive at $G_{1}=G_{2}$ : If two functions $F_{1}$ and $F_{2}$ satisfy (4) and if the corresponding operators $T_{F_{1}}$ and $T_{F_{2}}$ are isometrically equivalent and have the spaces $L_{p}[0, c]$ for all $c \in[0,1]$ as their only reducing manifolds-for example, if the functions $F_{j} \in D$-then $F_{1}=F_{2}$.

Observe that if our functions $F$ belong to $D$, then the similarity invariants of $T_{F}$, viz., $m, a$, and $c$, enter directly into the formulation of the isometry invariants (see $\mathrm{V}$ and [4] for similarity invariants). The "canonical functions" $F_{1}$ as given by (4) are the same for all $p$; however a given $T_{F}$ will have as its "canonical form" $T_{F_{1}}$ a transformation which in general does depend on $p$. If, for example, $F(x, y)$ 
$=1+2 x+i(x-y)$, then $m=1, a=1, c=2$. To describe its "canonical form" $F_{1}$ satisfying (4), it is convenient to introduce the function $K(x, y)=((8 x+1) /(8 y+1))^{1 / 2}$. A simple calculation shows that $F_{1}(x, y)=2 \exp (-i \log K)\left(K^{1 / q}+i\left(K^{1 / q}-K^{-1 / p}\right)\right)$.

\section{REFERENCES}

1. S. Banach, Thêorie des opérations linéaires, Warszawa, 1932.

2. M. S. Brodskir and M. S. Livšic, Spektral'nyi analiz nesamosopryažennyh operatorov i promežutočnye sistemy, Uspehi Mat. Nauk vol. 13 (1958) pp. 3-85.

3. G. K. Kalisch, On similarity, reducing manifolds, and unitary equivalence of certain Volterra operators, Ann. of Math. vol. 66 (1957) pp. 481-494.

4. - On similarity invariants of certain operators in $L_{p}$, Pacific J. Math., to appear.

5. L. A. Sahnovič, $O$ privedeniz vol'terrovskih operatorov $k$ prosteřemu vidu $i$ obratnyh sadačah, Izv. Akad. Nauk SSSR. Ser. Mat. vol. 21 (1957) pp. 235-262.

6. - Spektral'nyi analiz operatorov vida $K f=\int_{0}^{x} f(t) k(x-t) d t$, Izv. Akad. Nauk SSSR. Ser. Mat. vol. 22 (1958) pp. 299-308.

University of Minnesota

\section{THE NONEXISTENCE OF PROJECTIONS FROM $L^{1}$ TO $H^{1}$}

\section{J. NEWMAN}

Consider the Banach space $L^{1}(0,2 \pi)$ and the subspace $H^{1}$, of all functions all of whose negative Fourier coefficients vanish. It has been conjectured that $H^{1}$ has no complement in $L^{1}$, i.e., that $L^{1}$ is not the direct sum of $H^{1}$ and some other Banach space. In this note we give a proof of this conjecture.

The conjecture is of course equivalent to the following statement on projection operators.

THEOREM. There exists no bounded linear operator $P: L^{1} \rightarrow H^{1}$ for which $P f=$ f for all $f \in H^{1}$.

Proof. Suppose such a $P$ existed. Let $l_{n}(f)$ denote the $n$th Fourier coefficient of $P(f)$; then $l_{n}$ is a bounded linear functional on $L^{1}$ and as a result we have

$$
l_{n}(f(\theta))=\int_{0}^{2 \pi} f(\theta) \phi_{n}(\theta) d \theta,
$$

Received by the editors March 9, 1960. 